\title{
Collaborative Location estimation for confined spaces using Magnetic field and Inverse Beacon positioning
}

\author{
Mathangi Sridharan, Eliane Bodanese, John Bigham \\ School of Electronic Engineering and Computer Science \\ Queen Mary University of London \\ London, United Kingdom \\ Email: \{m.sridharan, eliane.bodanese, john.bigham\}@qmul.ac.uk
}

\begin{abstract}
A considerable amount of indoor positioning systems have been proposed for large-scale environments that extend over several metres. However, there has been less focus in designing an indoor localisation system for confined environments where the requirements of reliability and precision are high. The approach discussed in this paper employs a hybrid technique where Received Signal Strength Indicator (RSSI) fingerprinting using beacon and magnetic field sequential data are used in tandem to determine the zone where the person of interest is performing an activity. An offline location signature database is built for each of these methods where the RSSI and the magnetic field sequences are mapped with their respective walking routes or stationary positions within the household. We use the results of beacon RSSI fingerprinting as the basis to narrow down the searching space for magnetic field vector matching and later use Dynamic Time warping (DTW) algorithm to deduce the similarity between the measured and offline magnetic field sequences. We compare our approach with another variant of DTW known as Derivative Dynamic Time warping (DDTW) and evaluate the performance of each variant with and without the RSSI fingerprinting stage. To the best of our knowledge this is the first study of its kind that focuses on interpreting fine-grained deviation between positions or routes confined within a very small area.
\end{abstract}

Keywords-RSSI fingerprinting; beacon positioning; magnetic field matching; DTW; DDTW

\section{INTRODUCTION}

Indoor positioning systems have emerged as one of the most researched topics due to the fact that it can be used in a number of end user applications. Wireless technology and magnetic field matching have been used as independent solutions for indoor localisation in many past research works. However, both these technologies has its share of limitations when used as an individual solution for indoor positioning. Whilst the accuracy of the former method is affected by presence of obstructions and user movement, the latter suffers in accuracy when the number of samples in the reference database is low and when not updated regularly [1]. The magnetic field positioning method is also heavily dependent on user's orientation and velocity which makes it ineffective for finer grained interpretation when used as a solo solution for indoor positioning. In recent times, beacons have become increasingly popular as they consume less power and are inexpensive, but are mostly suited for providing proximity based solutions. Prior research suggests that when both these methods are used together, they complement each other and provide reliable results improving the overall accuracy [2,3].
Li et al. and Wang et al. use Wi-Fi fingerprinting to reduce the magnetic matching (MM) search space by limiting it to a circle around the estimated point from the Wi-Fi method $[2,3]$. The authors in [2] use dynamic time warping algorithm for magnetic field matching, while in [3], they employ a particle filter algorithm. Both these methods may not be suitable for confined spaces as the method for selecting the search area is based on only one estimated point during the Wi-Fi fingerprint matching stage. If a small radius is chosen for the MM search space, then there is a high possibility that the correct position is not part of the search area due to the high attenuation caused inside an indoor environment. Conversely, if the radius of the search space extends over few metres, the inclusion of Wi-Fi or Bluetooth Low Energy (BLE) method is nullified as the search space may consist of several overlapping routes and is likely to produce unfavourable results. In this study, we have developed a positioning system that overcome these issues and provides useful information to aid activity monitoring in a small household where distinguishing between points less than a metre poses a huge challenge. Our work is not focused on finding the $(\mathrm{x}, \mathrm{y})$ coordinates of the target but determining if the target is stationary at certain defined locations of interest, e.g., in the dining area, or moving along certain defined paths, e.g., kitchen to dining area.

The remainder of this paper is organised as follows. In Section II we explain the experimental setup, implementation of the two step fingerprinting process and its positioning algorithm. In Section III, we present the results of an activity centred case study and provide a performance analysis for a subsection of routes. Section IV concludes the paper.

\section{EXPERIMENTAL SETUP}

\section{A. Training stage Data Collection Process}

In this setup, a wearable beacon in the form of a pendant was chosen as the transmitter for the RSSI fingerprinting stage and a set of eight fixed Raspberry-Pis at known locations were used as receivers of the beacon's signal. This type of approach is referred to as inverse beacon positioning as the beacon is in motion, as opposed to the usual method of employing fixed beacons and a moving signal receiver. The location and number of Raspberry-Pis were chosen such that it provides comprehensive coverage over the entire flat and also based on the activities that need to be monitored. For measuring the three-dimensional magnetic field vector (MFV) sequence, a smartphone with its inbuilt magnetometer sensor was used that was positioned at chest level. One minute of RSSI and MFV

This research is partially supported by QMUL/Welcome Trust Institutional Strategic Support Fund through the LSI Digital Health Fund 
data was collected at each position marked by the Cartesian co-ordinates along a specific walking route or stationary position with length between each step measuring approximately $0.5 \mathrm{~m}$. The training database for both these techniques were populated by measuring the RSSI and MFV samples for conditions when a person is performing an activity with minimal movement (e.g., sitting in the dining area, sleeping in bed) and when a person walks along a certain path both in the forward and reverse direction (e.g., bedroom to bathroom, bathroom to bedroom). Due to the nature of the classification technique used during the training stage, sequences of continuous readings of RSSI and MFV are considered in this study over point-to-point estimation.

\section{B. Modelling of the Training Database}

The RSSI training database $\left(R S S I_{D B}\right)$ comprising of $\mathrm{N}$ routes or positions are of the format $R S S I_{D B}=\left\{S_{1}, \ldots, S_{N}\right\}$ where $S_{n}$ are the individual routes that belong to a specific walking route or a stationary position. Each route, $S_{n}$ will consist of a sequence of $M$ training samples modelled as $\left[\vec{L}_{i}, \overrightarrow{R S S}_{i}\right](1 \leq i \leq M)$ where $R S S_{\mathrm{i}}=\left\{P i_{R S S_{1}}, \ldots, P i_{R S_{8}}\right\}$ are the individual RSSI values of the beacon recorded at position co-ordinates $\vec{L}_{i}=\left(d_{x}^{i}, d_{y}^{i}\right)$.The database may contain duplicate readings for $\vec{L}_{i}$ since a sequence of RSSI values are registered for the same position. Similarly, the MFV training database contains sequential data where each point of the format $\left[\vec{L}_{i}, \overrightarrow{M F V}_{i}\right]$ consists of the 3-dimensional magnetic field vector, $\overrightarrow{M F V}_{i}=\left\{M F V_{x}, M F V_{y}, M F V_{z}\right\}$ recorded at position $\vec{L}_{i}=\left(d_{x}^{i}, d_{y}^{i}\right)$ along route $S_{n}$. The collective training database holds data for 43 different routes and positions covering all areas of the test environment.

\section{Implementation}

1) Stage 1 - RSSI Collection process using Beacon: One among the eight Raspberry-Pis is considered as the master and the rest will act as slaves. RSSI values of the beacon along

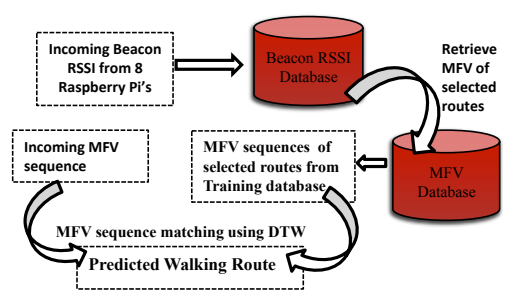

Fig. 1. Schematic Diagram of the Proposed System with its timestamp are collected by all the Raspberry-Pis and saved locally. The slave-Pis later transmit these files every few minutes to the master$\mathrm{Pi}$. The master-Pi is then in-charge of computing and sorting the data of all the devices including it's own data into a single file and transfers it to the indoor positioning program.

2) Stage 2 - MFV Collection process using Smart Phone: The MATLAB support package for Android sensors was used to collect magnetometer sensor data from the smart phone. The resulting data along with its timestamp was then used as input for the location estimation program implemented in MATLAB. A snapshot of the above discussed two stage fingerprinting process is illustrated in Fig. 1.

3) Description of the Positioning Algorithm: The offline collected beacon RSSI training data and online measured sequences are sorted based on the rank of their strongest signals. These ranked offline and online RSSI data are then compared against each other to find similarity between them and a list of corresponding matched routes and positions is returned. The routes considered for magnetic matching (MM) is reduced to 10 or less, which are selected as per the total number of occurrences and frequency of occurrence over the entire length of the measured sequence during the matching process in Stage-1. The magnetic signatures of these selected routes will alone be considered from the MFV training database for the following stage of MM fingerprinting. The next stage involves computing the similarity measure between the magnetic field vectors using 1-Nearest Neighbour algorithm with Dynamic Time Warping (DTW) as the distance measure. DTW is a well-known algorithm to measure the similarity between two sequential series of different lengths that vary in time or speed. It is chosen over Euclidean distance for time series measurement in most cases as the latter does not have the ability to perform one to many comparisons for time series of different lengths. DTW on the other hand, allows one to many mapping (compression and stretching) and is therefore suitable for comparing magnetic field sequences. We smooth the magnetometer sensor data of both the datasets using a low pass filter before measuring the similarity. The DTW algorithm then resorts to finding the predicted route that has the minimum distance measure from the warping matrix constructed with the offline and measured magnetic sequences.

\section{RESULTS AND ANALYSIS}

Fig. 2 illustrates the layout and placement of the 8 Raspberry-Pi's in a one bedroom flat which was chosen as the trial home to perform the study. An example case study is highlighted in Fig. 2 which shows the test results of the proposed method employing BLE and MFV fingerprinting against solo use of MFV fingerprinting when the user is performing a series of activities. The results from this case study prove that the predicted routes and positions by the proposed method is much closer to the actual results as compared to using only MFV sequences for location estimation. A high mismatch rate can be seen with the independent use of MFV fingerprinting method which are effectively eliminated when used along with a beacon.

To further evaluate the performance, the positioning error range was computed for 10 different test datasets for selected 15 routes within the trial home measured over different days. Additionally, we analysed the performance of the proposed method with a variant of DTW known as Derivative Dynamic Time Warping (DDTW) which makes use of the squared difference of the derivatives of times series under comparison [4]. Fig. 3 illustrates the performance comparison of BLE aided MM using DTW(BLE-MMDTW), BLE aided MM using DDTW(BLE-MMDDTW), MM using DTW(MMDTw) and MM using DDTW (MMDDTW) for the selected routes. The predicted distance range results of all the 15 routes from the 10 test datasets for these 4 methods are summarised in TABLE I. 

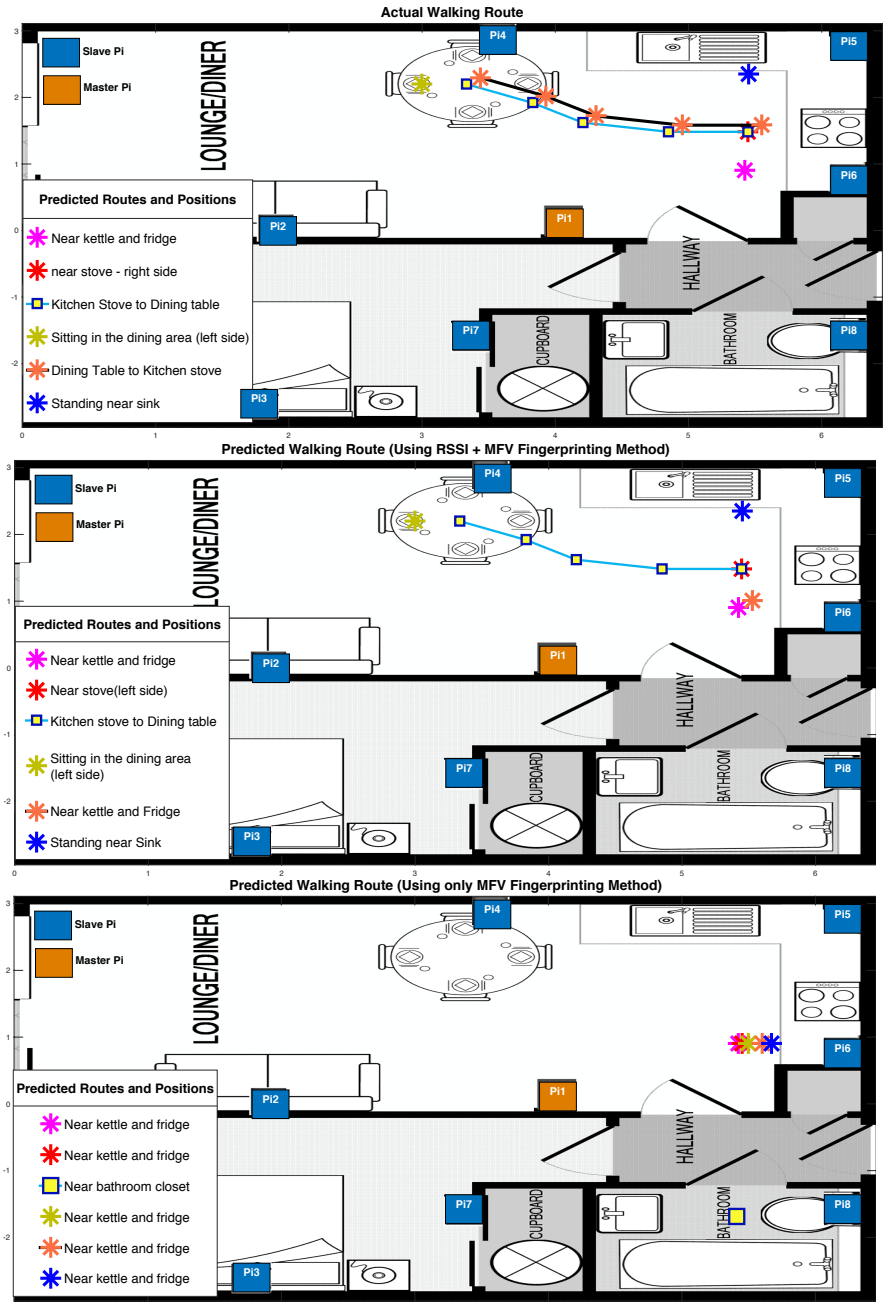

Fig. 2. Case Study Illustration when user performs the following activities [Uses the Fridge - Cooks using Stove - Moves to Dining Table from Stove - Sits in left side of the Dining area - Moves back to the Kitchen Stove - Uses the Kitchen Sink] (Note: The positions or routes specified in the legend are the results of each method, which are in the order of the activities performed by the user.

The test results shows that the prediction accuracy of BLEMM method using standard DTW and DDTW are relatively similar and is superior when compared to the other 2 methods with around 123 of the 150 routes (10 different replications of the 15 routes) falling within $1 \mathrm{~m}$ from the actual location. The results also reveal that the

TABLE I. OVERALL

COMPARISON OF PREDICTED

DISTANCE RANGE

\begin{tabular}{|c|c|c|c|c|}
\hline $\begin{array}{c}\text { Predicted } \\
\text { Distance Range } \\
\text { (in metres) }\end{array}$ & \multicolumn{4}{|c|}{ Number of Routes } \\
\cline { 2 - 5 } & $\begin{array}{c}\text { BLE- } \\
\text { MMDTW }\end{array}$ & $\begin{array}{c}\text { BLE- } \\
\text { MMDDTW }\end{array}$ & MMDTW & MMDDTW \\
\hline $\begin{array}{c}\text { Precise (exact } \\
\text { match) }\end{array}$ & 57 & 59 & 21 & 17 \\
\hline $\begin{array}{c}\text { Very Near } \\
\text { (m) }\end{array}$ & 66 & 63 & 30 & 7 \\
\hline Near $<2 \mathrm{~m})$ & 11 & 10 & 23 & 16 \\
\hline Far $(<3 \mathrm{~mm})$ & 6 & 6 & 19 & 25 \\
\hline Very Far (>3m) & 10 & 12 & 57 & 85 \\
\hline
\end{tabular}

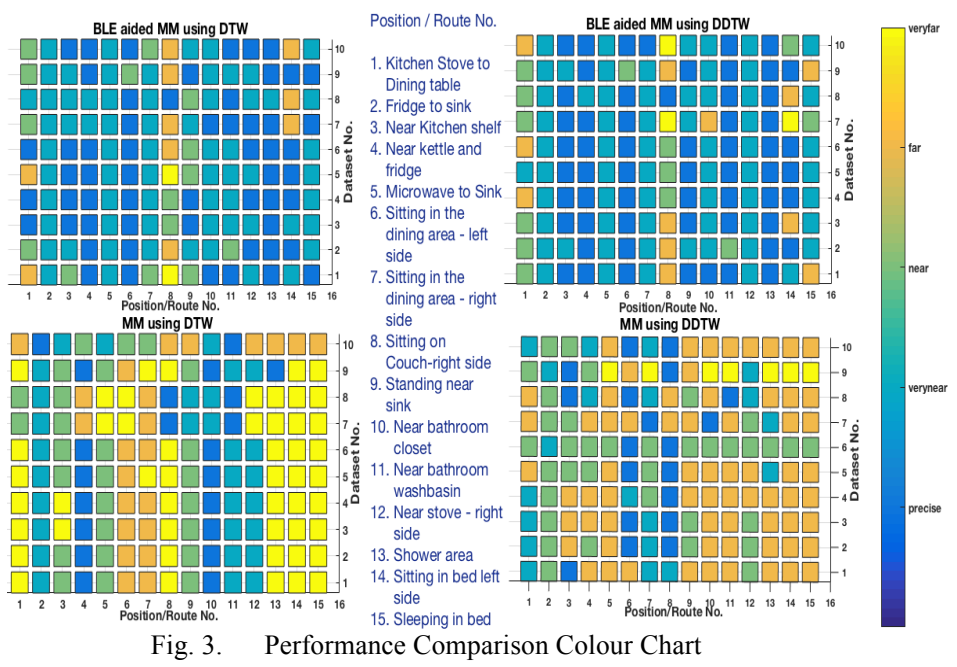

However, the prediction of MM method is better over BLE-MM method when the person is sitting on the couch which may be due to the incorrect placement of Raspberry-Pi receiver no.2 (Refer Fig. 2) behind the couch as the human body is more likely to obstruct the beacon signal. The future work will address this limitation by changing the placement of this Raspberry-Pi receiver to a suitable Line of Sight (LoS) position.

\section{CONCLUSION}

In order to overcome the shortcomings of beacon signal stability and mismatch issues in magnetic field fingerprinting, this paper presents a hybrid method for localisation in confined spaces by using inverse beacon fingerprinting method to narrow down the magnetic field vector matching space. The process of amalgamation of results from both the techniques contributes to higher performance. The empirical results demonstrate that our proposed method has high potential in improving accuracy when compared to the independent use of magnetic matching techniques. This method was developed as part of research for activity monitoring in a household where the focus was to maintain a minimal sensing environment and also to help determine the length of sensor events to be considered for differentiation between individual activities. Future work will concentrate on reducing the number of Raspberry-Pis and on using a single wearable embedded with beacon, magnetometer and other accompanying sensors such as accelerometer and gyrometer to differentiate if the person is stationary or walking.

\section{REFERENCES}

[1] Torres-Sospedra, Joaquín, et al. "Providing Databases for Different Indoor Positioning Technologies: Pros and Cons of Magnetic Field and Wi-Fi Based Positioning." Mobile Information Systems, 2016.

[2] Y. Li, Y. Zhuang, H. Lan, P. Zhang, X. Niu, N. El-Sheimy, "Wi-Fiaided magnetic matching for indoor navigation with consumer portable devices", Micromachines, vol. 6, no. 6, pp. 747-764, 2015.

[3] E.Wang, M.Wang, Z.Meng, X.Xu. "A Study of WiFi-Aided Magnetic Matching Indoor Positioning Algorithm." Journal of Computer and Communications 5.03, pp. 91-101, 2017.

[4] E. J. Keogh and M. J. Pazzani, "Derivative dynamic time warping," in Proceedings of the 2001 SIAM International Conference on Data Mining. SIAM, 2001, pp. 1-11 